\title{
MULTIPLE NONTRIVIAL SOLUTIONS OF RESONANT AND NONRESONANT ASYMPTOTICALLY LINEAR PROBLEMS
}

\author{
SHAIR AHMAD ${ }^{1}$
}

\begin{abstract}
We give simple conditions under which a second order semilinear elliptic boundary value problem with the zero solution has at least two nonzero solutions. Our conditions involve the change in the spectrum of the linearization of the problem going from zero to infinity.
\end{abstract}

Let $\Omega$ be a smooth bounded domain in $\mathbf{R}^{n}(n \geq 1)$ and $g$ be a $C^{1}$ scalar function defined on $\mathbf{R}$ such that $g(0)=0$. We consider the boundary value problem

$$
\Delta u+g(u)=0 \quad \text { in } \Omega, \quad u \mid \partial \Omega=0
$$

and investigate the existence of nonzero solutions. Let $0<\lambda_{1}<\lambda_{2} \leq \cdots \leq \lambda_{m} \leq$ $\lambda_{m+1} \leq \cdots$ denote the increasing sequence of eigenvalues of the linear problem

$$
\Delta u+\lambda u=0 \quad \text { in } \Omega, \quad u \mid \partial \Omega=0 .
$$

It follows from an abstract result due to Amann and Zehnder [2] (see [6] for a simplified proof) that if

$$
g^{\prime}(0)=a, \quad \lim _{|s| \rightarrow \infty} g(s) / s=b,
$$

neither $\underline{a}$ nor $b$ is an eigenvalue of problem (2), and there is some eigenvalue between $\underline{a}$ and $b$, then there exists a nonzero solution of (1).

In this note we give conditions under which (1) has at least two nontrivial solutions. We consider the cases $b \in\left(\lambda_{1}, \lambda_{2}\right), a<\lambda_{1}$ and the resonance case $b=\lambda_{1}$. Our main tools are recent theorems of Ambrosetti [4] and Hofer [7]. These theorems have been combined in $[8]$ to establish existence of multiple solutions of a nonhomogeneous problem with jumping nonlinearities. The variational approach used to study the resonance case is similar to that first used in [1 and 10].

We remark that the subsolution-supersolution method (see [12]) can be used to establish the existence of a positive and a negative solution if $b<\lambda_{1}<a$.

THEOREM 1. If $\lambda_{1}<b<\lambda_{2}$ and either $a<\lambda_{1}$ or $a \in\left(\lambda_{m}, \lambda_{m+1}\right)$ for some $m \geq 2$, then (1) has at least two nontrivial solutions. The same is also true if $a<\lambda_{1}$ and $b \in\left(\lambda_{m}, \lambda_{m+1}\right)$ for some $m \geq 1$.

Proof. Let $G^{\prime}(\xi)=g(\xi)$ for all $\xi \in \mathbf{R}$ and $G(0)=0$. Let $H$ denote the Sobolev space $W_{1,2}^{0}(\Omega)$ with inner product

$$
\langle u, v\rangle=\int_{\Omega}(\nabla u, \nabla v) d x
$$

Received by the editors February 25, 1985.

1980 Mathematics Subject Classification. Primary 34B15, 58E05.

Key words and phrases. Critical point of mountain-pass type, Leray-Schauder degree, eigenvalues, nondegenerate critical point, Landesman-Lazer condition.

${ }^{1}$ This work was partially supported by Sonderforschungsbereich 72 at the University of Bonn. 
where (, ) is the $\mathbf{R}^{n}$-inner product. If $f: H \rightarrow \mathbf{R}$ is defined by

$$
f(u)=\int_{\Omega}\left(|\nabla u|^{2} / 2-G(u)\right) d x
$$

then standard arguments $[\mathbf{1 1}]$ show that $f \in C^{2}$,

$$
f^{\prime}(u)(v)=\int_{\Omega}((\nabla u, \nabla v)-g(u) v) d x
$$

for $u, v \in H$, and for $u, v, w \in H$,

$$
f^{\prime \prime}(u)(v)(w)=\int_{\Omega}\left((\nabla v, \nabla w)-g^{\prime}(u) v w\right) d x .
$$

We see that weak solutions of $(1)$ coincide with critical points of $f$ and, by regularity theory, weak solutions are smooth. By the Riesz-Frechet theorem, the Sobolev imbedding theorem, (4) and (6) we can write $f^{\prime}(u)(v)=\langle u, v\rangle-\langle N(u), v\rangle$, where $N: H \rightarrow H$ is continuous and compact. Let $T: H \rightarrow H$ be the compact selfadjoint operator defined implicitly by $\langle T u, v\rangle=(b u, v)_{0}$ where $(,)_{0}$ is the $L^{2}(\Omega)$-inner product. Our assumptions on $g$ imply that

$$
\|T u-N(u)\| /\|u\| \rightarrow 0
$$

as $\|u\| \rightarrow \infty$, where \|\| is the norm in $H$. Since $b$ is not an eigenvalue of problem (2), the mapping $I-T$ is invertible and consequently there exists a number $\delta>0$ such that $\|u-T u\| \geq 2 \delta\|u\|$ for all $u \in H$. By (8) there exists $r>0$ such that $\|u-N(u)\| \geq \delta\|u\|$ if $\|u\| \geq r$. It follows that if $\left\{u_{n}\right\}_{1}^{\infty}$ is a sequence in $H$ such that $u_{n}-N\left(u_{n}\right) \rightarrow 0$ in $H$ as $n \rightarrow \infty$, then $\left\{u_{n}\right\}_{1}^{\infty}$ is bounded and hence, by compactness of $N$, some subsequence of $\left\{u_{n}\right\}_{1}^{\infty}$ converges. This shows that $f$ satisfies the well-known Palais-Smale condition.

Assume that the first set of conditions of the theorem holds. By l'Hôpital's rule, $G(\xi) / \xi^{2} \rightarrow b / 2$ as $|\xi| \rightarrow \infty$. Therefore, there exist constants $c_{1}, c_{2}, d_{1}$ and $d_{2}$ such that $\lambda_{1}<c_{1}<c_{2}<\lambda_{2}$, and for all $\xi \in \mathbf{R}$,

$$
c_{1} \xi^{2} / 2-d_{1} \leq G(\xi) \leq c_{2} \xi^{2} / 2+d_{2} .
$$

If $\phi_{1}$ is a normalized eigenfunction corresponding to $\lambda_{1}$ and $Y$ denotes the subspace of $H$ consisting of functions orthogonal to $\phi_{1}$ in $L^{2}(\Omega)$, then $\int_{\Omega}|\nabla y|^{2} d x \geq$ $\lambda_{2} \int_{\Omega} y^{2} d x$ for all $y \in Y$. Hence, by (5), if $y \in Y, f(y) \geq 1 / 2\left[\lambda_{2}-c_{2}\right]|y|_{0}^{2}-$ $d_{2}($ meas $\Omega) \geq c^{*}$, where $c^{*}=-d_{2}($ meas $\Omega)$ and ||$_{0}$ is the $L^{2}(\Omega)$-norm. Since $\left\|\phi_{1}\right\|^{2}=\lambda_{1}|\phi|_{0}^{2}$, it follows from (5) and (9) that $f\left(s \phi_{1}\right) \leq s^{2} / 2\left[\lambda_{1}-c_{1}\right]+d_{1}($ meas $\Omega)$; so $f\left(s \phi_{1}\right) \rightarrow-\infty$ as $|s| \rightarrow \infty$. Let $s_{0}$ be so large that $f\left( \pm s_{0} \phi_{1}\right)<c^{*}$ and set $v_{0}=-s_{0} \phi_{1}, v_{1}=s_{0} \phi_{1}$. Let $A$ consist of all continuous maps $\alpha:[0,1] \rightarrow H$ such that $\alpha(0)=v_{0}$ and $\alpha(1)=v_{1}$. If $\alpha \in A$, then $\alpha(\bar{t}) \in Y$ for some $\bar{t} \in[0,1]$ and consequently, $c_{0} \equiv \inf _{\alpha \in A} \max _{t \in[0,1]} f(\alpha(t)) \geq c^{*}$. By a slight variation of the proof of the well-known Ambrosetti-Rabinowitz mountain pass theorem (see $[3,4,11]$ ) we infer the existence of $u_{0}$ in $H$ such that $f^{\prime}\left(u_{0}\right)=0$ and $f\left(u_{0}\right)=c_{0}$.

If $f^{-1}\left(c_{0}\right)=\left\{u_{0}\right\}$ and $\left\{u_{0}\right\}$ is a nondegenerate critical point of $f$, i.e. if there exists no $v \neq 0$ in $H$ such that $f^{\prime \prime}\left(u_{0}\right)(v)(w)=0$ for all $w \in H$, then a recent result of Ambrosetti [4, p. 19] shows that the Morse index of $u_{0}$ is 1 . (This is the maximum integer $k$ such that the quadratic form $Q(v)=f^{\prime \prime}\left(u_{0}\right)(v)(v)$ is negative definite on some $k$-dimensional subspace of $H$.) From (3) and (7) we see that 
$f^{\prime \prime}(0)(v)(w)=\int_{\Omega}((\nabla v, \nabla w)-a v w) d x$ for $v, w \in H$, and therefore, since $a$ is not an eigenvalue of (2), 0 is a nondegenerate critical point of $f$. If $a<\lambda_{1}$, then for $v \neq 0$

$$
f^{\prime \prime}(0)(v)(v)=\|v\|_{0}^{2}-a|v|_{0}^{2} \geq\left(\lambda_{1}-a\right)|v|_{0}^{2}>0
$$

so that the Morse index of 0 is 0 . If $a \in\left(\lambda_{m}, \lambda_{m+1}\right)$ for some $m \geq 2$ and $V$ denotes the subspace of $H$ spanned by the eigenfunctions corresponding to eigenvalues $\lambda_{k}$ of (2) with $k \leq m$, then $f^{\prime \prime}(0)(v)(v) \leq\left(\lambda_{m}-a\right)|v|_{0}^{2}<0$ if $v \in V$ and $v \neq 0$; thus the Morse index of 0 is at least 2. Hence, by Ambrosetti's theorem there exists $u_{0}, u_{0} \in f^{-1}\left(c_{0}\right)$, with $u_{0} \neq 0$ and $f^{\prime}\left(u_{0}\right)=0$.

If $T$ is the compact, selfadjoint operator defined above, then since $I-T$ is invertible, the Leray-Schauder degree $d\left(I-T, B_{R}, 0\right)$ is defined if $B_{R}$ is the ball of radius $R$ centered at 0 in $H$. If $T v=\gamma v$ for some $v \neq 0$, then for all $w \in H$, $\int_{\Omega}(\gamma(\nabla v, \nabla w)-b v w) d x=0$. Hence, $b / \gamma$ is an eigenvalue of $(2)$, and $\gamma=b / \lambda_{m}$ for some $m$. Thus, since $\lambda_{1}<b<\lambda_{2}, T$ has exactly one eigenvalue bigger than 1 , and it follows that $d\left(I-T, B_{R}, 0\right)=-1$ (see, for example, [9, p. 66]).

From (8) it follows that for $R$ sufficiently large, $\operatorname{deg}\left(I-N, B_{R}, 0\right)$ $=\operatorname{deg}\left(I-T, B_{R}, 0\right)=-1$. The same type of argument used to calculate $d\left(I-T, B_{R}, 0\right)$ shows that the Leray-Schauder index of 0 as a zero of $u-N(u)$ is 1 if $a<\lambda_{1}$ and $(-1)^{m}$ if $a \in\left(\lambda_{m}, \lambda_{m+1}\right)$ for some $m \geq 2$.

If 0 and $u_{0}$ were the only critical points of $f$, then a recent result of Hofer [7] would imply that the Leray-Schauder index of $u_{0}$ as a zero of $u-N(u)$ equals -1 . But this is impossible since $d\left(I-N, B_{R}, 0\right)$, for $R$ large, is the sum of the L-S indices of 0 and $u_{0}$. This contradiction proves the first part of the theorem.

To prove the second part, we note that the condition $a<\lambda_{1}$ implies that $f$ has a strict local minimum at $u=0$ since $f^{\prime}(0)=0$ and for $v \in H, f^{\prime \prime}(0)(v)(v)=$ $\|v\|^{2}-a|v|_{0}^{2} \geq k\|v\|^{2}$, where $k=1-a / \lambda_{1}>0$. The condition $b \in\left(\lambda_{m}, \lambda_{m+1}\right)$ for some $m>1$ implies the existence of constants $c_{3}>\lambda_{1}$ and $d_{3}$ such that $G(\xi) \geq$ $\frac{1}{2} c_{3} \xi^{2}-d_{3}$. Therefore, from (5), we have $f\left(s \phi_{1}\right) \leq \frac{1}{2} s^{2}\left[\lambda_{1}-c_{3}\right]+d_{3}($ meas $\Omega) \rightarrow-\infty$ as $s \rightarrow \infty$. By the ordinary mountain pass theorem [3], it follows that $f$ has a critical point $u_{0}$ with $f\left(u_{0}\right)>f(0)=0$. The L-S index of 0 as a zero of $u-N(u)$ is 1 , and the dgree of $I-N$ with respect to $B_{R}$ and 0 is $(-1)^{m}$ if $R$ is sufficiently large. If $u_{0}$ and 0 were the only critical points of $f$, then, by Hofer's theorem [7], the L-S index of $u_{0}$ would be -1 . But this would imply that for $R$ large, $(-1)^{m}=d\left(I-N, B_{R}, 0\right)=1-1=0$, a contradiction. This proves the result.

We indicate briefly how the methods used to prove Theorem 1 can be used to obtain multiple nontrivial solutions of the resonance-type problem

$$
\Delta u+\lambda_{1} u+g_{0}(u)=0, \quad u \mid \partial \Omega,
$$

where the function $g_{0}$ is bounded. The existence of solutions of $(10)$ when $g_{0}(0)=0$ has recently been investigated by Bartolo, Benci and Fortunato in [5]. The following theorem appears to be a new result for this type of problem.

THEOREM 2. Let $g_{0}$ be a $C^{1}$ function such that $g_{0}(\xi)$ is bounded for $\xi \in$ $(-\infty, \infty)$ and $g_{0}(0)=0$. If

$$
\limsup _{\xi \rightarrow-\infty} g(\xi)<0<\liminf _{\xi \rightarrow+\infty} g(\xi)
$$

and either $g_{0}^{\prime}(0)<0$ or there exists $m \geq 2$ such that $\lambda_{m}<g_{0}^{\prime}(0)+\lambda_{1}<\lambda_{m+1}$, then problem (10) has at least two nontrivial solutions. 
PROOF. Let $G_{0}^{\prime}(\xi)=g_{0}(\xi)$ and $G_{0}(0)=0$. Let $\phi_{1}$ be the normalized positive eigenfunction corresponding to $\lambda_{1}$, let $Y$ be as in the proof of Theorem 1, and let $f$ be as in (5) with $G(\xi)=\lambda_{1} \xi^{2} / 2+G_{0}(\xi)$. Condition (11) implies that $f\left(s \phi_{1}\right)=-\int_{\Omega} G\left(s \phi_{1}(x)\right) d x \rightarrow-\infty$ as $|s| \rightarrow \infty$. Therefore, by Lemma 2.8 of [10] $f$ satisfies P-S and $f$ is bounded below on $Y$ (see Remark 2.8 of [10]). If $s_{0}$ is chosen so that $f\left( \pm s_{0}\right)<c^{*}$, where $c^{*}$ is the infimum of $f$ on $Y$, and $A$ and $c_{0}$ are defined as in the proof of Theorem 1, then the same argument used there gives the existence of $u_{0}$ such that $f^{\prime}\left(u_{0}\right)=0$ and $f\left(u_{0}\right)=c_{0}$. The proof of Theorem 1 shows that if $g^{\prime}(0)<0$, then the Morse index of 0 as a critical point of $f$ is 0 , and if $\lambda_{m}<g^{\prime}(0)+\lambda_{1}<\lambda_{m+1}$ for some $m \geq 2$, then the Morse index of zero is $m$. Therefore Ambrosetti's theorem implies the existence of a critical point $u_{0} \in f^{-1}\left(c_{0}\right)$ with $u_{0} \neq 0$.

The proof of Theorem 1 will imply the existence of at least three critical points of $f$ if it can be shown that the degree of $I-N$ with respect to $B_{R}$ and 0 is -1 for $R$ large where $N$ is defined as before with $g(\xi)=\lambda_{1} \xi+g_{0}(\xi)$. Let $\gamma>0$ be chosen so that $\lambda_{1}+\gamma<\lambda_{2}$, and let $g(\xi, t)=g(\xi)+\gamma t$ for $0 \leq t \leq 1$. Since $g(\xi, 0)=\lambda_{1}+g_{0}(\xi)$ and $g(\xi, 1) / \xi \rightarrow \lambda_{1}+\gamma \in\left(\lambda_{1}, \lambda_{2}\right)$ as $|\xi| \rightarrow \infty$, to show that $d\left(I-N, B_{R}, 0\right)=-1$ for large $R$, it is sufficient to establish an a priori bound in $H$ of solutions of

$$
\Delta u+g(u, t)=0, \quad u \mid \partial \Omega=0,
$$

independent of $t$ in $[0,1]$. The result then follows by the homotopy invariance of the degree. Since $(-\Delta)^{-1}$ may be regarded as a compact mapping from $L^{p}(\Omega)$ onto $W_{2, p}(\Omega) \cap W_{1,2}^{0}(\Omega) \subset C^{1}(\bar{\Omega})$ for $p>N$, it is enough to establish an a priori $L^{\infty}(\Omega)$ bound on solutions of $(12)$ independent of $t$ in $[0,1]$. Assuming that no such bound exists, there exists a sequence of numbers $\left\{t_{n}\right\}_{1}^{\infty}$ in $[0,1]$ and a corresponding sequence of smooth functions $\left\{u_{n}\right\}_{1}^{\infty}$ such that $u_{n}$ is a solution of (12) when $t=$ $t_{n}$ and $\left|u_{n}\right|_{\infty} \rightarrow \infty$ as $n \rightarrow \infty$. We may assume $t_{n} \rightarrow t_{0} \in[0,1]$ as $n \rightarrow \infty$. Let $v_{n}=u_{n} /\left|u_{n}\right|_{\infty}$. Since $-\Delta v_{n}=\lambda_{1} v_{n}+\gamma t_{n} v_{n}+g_{0}\left(u_{n}\right) /\left|v_{n}\right|_{\infty}$ is bounded in $L^{\infty}(\Omega)$ independently of $n$, by compactness and bootstrapping, we may assume that $\left\{v_{n}\right\}_{1}^{\infty}$ converges in $C^{1}(\bar{\Omega})$ to a smooth function $v$ such that $\Delta v+\left(\lambda_{1}+\gamma t_{0}\right) v=0$, $v \mid \partial \Omega=0$ and $|v|_{\infty}=1$. By choice of $\gamma$ we must have $t_{0}=0$ and $v=c \phi_{1}$ for some $c \neq 0$. We consider the case $c>0$-the other case is treated similarly. Since $\phi_{1}$ is strictly positive on $\Omega$ and its normal derivative is strictly negative on $\partial \Omega, u_{n}(x) \rightarrow \infty$ everywhere on $\Omega$, and $u_{n}(x)>0$ everywhere on $\Omega$ for $n$ large. Integrating $\phi_{1} \Delta u_{n}+\phi_{1} \lambda_{1} u_{n}+\phi_{1}\left(\gamma t_{n} u_{n}+g_{0}\left(u_{n}\right)\right)=0$ over $\Omega$, integrating the first term twice by parts, and using $\Delta \phi_{1}+\lambda_{1} \phi_{1}=0, \phi_{1}\left|\partial \Omega=u_{n}\right| \partial \Omega=0$, we obtain $\int_{\Omega} \phi_{1}\left(\gamma t_{n} u_{n}+g_{0}\left(u_{n}\right)\right) d x=0$ for all $n$. But for $n$ large, $\int_{\Omega} \phi_{1}\left(\gamma t_{n} u_{n}+g_{0}\left(u_{n}\right)\right) d x \geq$ $\int_{\Omega} \phi_{1} g_{0}\left(u_{n}\right) d x$, and by Fatou's lemma and (11),

$$
\liminf _{n \rightarrow \infty} \int_{\Omega} \phi_{1}(x) g_{0}\left(u_{n}(x)\right) d x \geq \int_{\Omega} \liminf _{n \rightarrow \infty} \phi_{1}(x) g_{0}\left(u_{n}(x)\right) d x>0,
$$

so we have a contradiction. By earlier remarks, this proves the theorem.

We note that (11) is the usual Landesman-Lazer condition sufficient for solvability of (12) for smooth bounded $g_{0}$ not necessarily satisfying $g_{0}(0)=0$. 


\section{REFERENCES}

1. S. Ahmad, A. Lazer and J. Paul, Elementary critical point theory and perturbations of elliptic boundary value problems at resonance, Indiana Univ. Math. J. 25 (1976), 933-944.

2. H. Amann and E. Zehnder, Nontrivial solutions for a class of nonresonance problems and applications to nonlinear differential equations, Ann. Scuola Norm. Sup. Pisa 7 (1980), $539-603$.

3. A. Ambrosetti and P. H. Rabinowitz, Dual variational methods in critical point theory and applications, J. Functional Anal. 14 (1973), 349-381.

4. A. Ambrosetti, Differential equations with multiple solutions and nonlinear functional analysis, Equadiff 82, Lecture Notes in Math., vol. 1017, Springer-Verlag, Berlin and New York, 1983.

5. P. Bartolo, V. Benci and D. Fortunato, Abstract critical point theorems and applications to some nonlinear problems with "strong" resonance at infinity, Nonlinear Anal. 7 (1983), 981-1012.

6. C. C. Chang, Solutions of asymptotically linear operator equations via Morse theory, Comm. Pure Appl. Math. 34 (1981), 693-712.

7. H. Hofer, $A$ note on the topological degree at a critical point of mountain pass type, Proc. Amer. Math. Soc. 90 (1984), 309-315.

8. A. C. Lazer and P. J. McKenna, Critical point theory and boundary value problems with nonlinearities crossing multiple eigenvalues, Comm. Partial Differential Equations 10 (1985), 107-150.

9. L. Nirenberg, Topics in nonlinear functional analysis, Courant Inst. of Math. Sciences, New York, 1974.

10. P. H. Rabinowitz, Some minimax theorems and applications to nonlinear partial differential equations, Nonlinear Analysis (L. Cesari, R. Kannan and H. F. Weinberger, eds.), Academic Press, New York, 1978, pp. 161-177.

11. _ The mountain pass theorem: theme and variations, Differential Equations, Lecture Notes in Math., Springer-Verlag, Berlin and New York, 1982.

12. D. H. Sattinger, Topics in stability and bifurcation theory, Lecture Notes in Math., vol. 309, Springer-Verlag, Berlin and New York, 1973.

Department of Mathematics and Computer SCience, University of Miami, CORAL GABLES, FLORIDA 33124 